\title{
Molecular imaging of stem cell therapy in brain tumors: a step towards personalized medicine
}

Nora Sandu ${ }^{1}$, Bernhard Schaller ${ }^{2}$

1Department of Neurosurgery, University of Lausanne, Switzerland

2University of Oradea, Romania

Submitted: 12 August 2010

Accepted: 14 December 2010

Arch Med Sci 2012; 8, 4: 601-605

DOI: 10.5114 /aoms.2012.30282

Copyright $\odot 2012$ Termedia \& Banach

Advances in gene-based medicine since the 1990s have ushered in a new therapeutic strategy of gene therapy for inborn error genetic diseases and also for cancer [1-6]. Personalized treatment strategies using stem, modified or also genetically engineered cells are becoming a reality in clinical medicine. Allogenic or autologous cells can be used for treatment and possibly for early diagnosis of diseases. Hematopoietic, stromal and organ specific stem cells are under evaluation for cell-based therapies, not only for neurological, but also cardiac, pneumological, gynecological, autoimmune and other disorders. Such cell replacement therapy and gene transfer have provided the basis for the development of potentially powerful new therapeutic strategies for a broad spectrum of neurological diseases. However, currently available treatment modalities for brain tumors, including radical surgical resection followed by radiation and chemotherapy, have substantially improved the survival rates [7, 8]; however, a large proportion of patients with brain tumors remain incurable [9]. Therefore, there is substantial need for more effective therapies for patients with malignant brain tumors, and gene therapy targeting brain tumors should fulfill this requirement. Gene therapy for brain tumors includes many therapeutic strategies and these strategies can be subgrouped in two major categories: (i) molecular and (ii) immunologic. The widely used molecular gene therapy approach is suicide gene therapy based on the conversion of non-toxic prodrugs into active anticancer agents via introduction of enzymes, and genetic immunotherapy involves the gene transfer of immune-stimulating cytokines including interleukin (IL)-4, IL-12 and tumor necrosis factor related apoptosis inducing ligand (TRAIL) [10, 11]. For both molecular and immune gene therapy, stem cells can be used as a delivery vehicle of therapeutic genes [12]. Stem cells possess an inherent tumor tropism and have the capacity to target therapeutic genes to tumors, which supports their use as a reliable delivery vehicle to target therapeutic gene products to primary brain tumors and metastatic cancers throughout the brain $[13,14]$. The main promise of this emerging technology centers on the potent migratory tropism exhibited by stem cells for disseminated foci of intracranial pathologic findings. This important characteristic, which has been validated in a wide set of preclinical studies, forms a foundation for the use of transplanted stem cell populations as vehicles for the delivery of tumor-toxic molecules to sites of intracranial tumor $[9,14]$. The significance of neuronal stem cell (NSC)-based gene therapy for brain tumor is that it is possible to exploit the tumor-tropic
Corresponding author: Bernhard Schaller MD, PhD, DSC

University of Oradea

Romania

Phone: 07966699 42b

E-mail:

skull_base-surgery@yahoo.de 
property of stem cells to mediate effective, tumorselective therapy for primary and metastatic cancers in the brain and outside, for which no tolerated curative treatments are currently available. Before widely using stem cells in clinical trials, translational research in experimental animal models is needed, with a critical emphasis on developing noninvasive methods for tracking the temporal and spatial homing of these cells to target tissues.

The current development of novel treatment strategies for brain tumors based on the transplantation or infusion of cells that can seek out invading tumor cells demands thorough in vivo monitoring [7]. Molecular imaging may allow modification of treatment to maximize treatment efficacy and minimize late effects on chemotherapy and radiotherapy. Especially, stem cells attract great interest as they show a tropism to tumor cells and will even migrate long distances to track down single tumor cells $[15,16]$. It is thought that the migration of stem cells to neoplastic cells is mediated by the secretion of chemical factors, such as vascular endothelial growth factor (VEGF) [7, 17], that are involved in the proliferation, growth, and maintenance of tumors. Transplantation of unaltered stem cells has resulted in a prolonged survival of animals with experimental tumors [18], but the insertion of anti-tumor cytokine genes (e.g., IL-12) or proapoptotic genes (e.g., TRAIL) further improves the efficiency of this approach [19]. Little is known about how these cells exert their beneficial effects in vivo. Despite contrary evidence from preclinical studies, there is some concern that transplantation of stem cells could further exacerbate tumor formation as there is mounting evidence that brain tumors are potentially caused by a single stem cell that did not differentiate [20]. Targeting oncogenic pathways that are essential to the survival and growth of brain tumor stem cells represents a promising area for developing therapeutics. However, due to the multiple oncogenic pathways involved in brain tumors, it is necessary to determine which pathways are the essential targets for therapy.

The identification of genetic and biochemical mechanisms underlying tumor growth and progression along with the unraveling of the human genome provided a plethora of new targets for cancer detection, treatment and monitoring. Simultaneously, the extraordinary development of a number of imaging technologies, including hybrid systems, allowed the visualization of biochemical, molecular and physiological aberrations linked to underlying mutations in a given tumor. In vivo evaluation of complex biological processes such as proliferation, apoptosis, angiogenesis, metastasis, gene expression, receptor-ligand interactions, transport of substrates and metabolism of nutrients in human cancers is feasible by molecular imaging using positron emission tomography/computed tomography (PET/CT) and radiolabeled molecular probes. Some of these compounds are in preclinical phases of evaluation whereas others have already been applied in clinical settings. Molecular imaging of brain tumors provides a prominent example of how some biological processes and target expression can be visualized by PET/CT in animal tumor models and also in patients with various cancers - such as lung, breast or even brain tumors - for the noninvasive detection of well-known markers of tumor aggressiveness, invasiveness and resistance to treatment and for the evaluation of tumor response to therapy while reducing side effects or even for evaluation of prognosis. Such ability to monitor cell therapy in vivo is therefore desirable to potentially provide more control over the activity of stem cells $[21,22]$. This strategy is relatively new for brain tumors, but is already well established for other cancers [23]. One possibility is that stem cells will be engineered with a suicide gene [24] that could be activated if transplanted cells do not behave in a therapeutic manner. However, the ability to control the activity of transplanted cells presupposes that it is possible to visualize the presence, location, and activity of stem cells in vivo. Although magnetic resonance imaging (MRI) has been extensively used to track cells repeatedly in vivo [25], it is the higher specificity of positron emission tomography (PET) ligands and its ability to detect reporter genes that are attractive features to develop longterm in vivo monitoring of transplanted cells [26]. This new "image and treat" strategy, involving assessment of target presence and distribution in an individual patient followed by optimized, targetspecific drug delivery, may potentially improve efficacy of brain tumors while reducing side effects. However, this strategy is a great step towards a personalized medicine.

The reporter gene approach has also been used with MRI to facilitate iron uptake $[27,28]$. The contrast agents used to detect transplanted cells from the background of the brain are incorporated into the cell in vitro before transplantation [22, 29]. The presence of the contrast agent inside the cell therefore allows the prolonged visualization of grafted cells on MRI scans. However, the continued presence of the contrast agent inside the cell can also affect cellular functions $[25,30]$. Although this approach has been demonstrated with PET/single photon emission computed tomography (SPECT) ligands and allowed monitoring for up to 14 days [31], it is the short half-life of PET ligands that compromises the long-term visualization of cells. A more promising method is to engineer reporter genes inside the cells before grafting and to systemically inject the PET ligands to detect transplanted cells [32]. The use of reporter genes avoids 
most issues pertaining to the long-term effects the contrast agent might exert on cellular functions. The repeated application of a contrast agent complements a flexible imaging approach that can target various relevant targets, such as fludeoxyglucose (FDG)-PET to investigate tumor metabolism $[29,33,34]$. Especially for the continued assessment of tumor evaluation in response to treatment, it is important to be able to assess the malignancy of the tumor noninvasively at various time points. The main concerns with this approach regard the potential immunogenic properties of reporter genes that might lead to graft rejection or the down-regulation of the reporter gene abolishing graft detection. Unfortunately, at present little research is dedicated to the development of PET techniques that would allow the continued assessment of cell therapy. The use of radioligands to a reporter gene will provide maximum flexibility and further integration into a wider molecular imaging strategy of brain tumors. Currently, many studies utilize the combination of two or more reporter genes that would enable the use of different imaging modalities to overcome the drawbacks associated with a single reporter gene and/or associated detection system [35].

Apart from in vivo monitoring, PET will also be instrumental to gain a greater mechanistic understanding of how cell therapy exerts its therapeutic effects. As stem cells can be engineered to express particular genes and serve as Bsmart delivery vehicles [36], their effects on tumor cells could be through inhibition of angiogenesis, induction of apoptosis in tumor cells, or induction of differentiation of tumor cells $[37,38]$. Being able to study how stem cells will alter the molecular composition of the tumor will allow in vivo monitoring of the therapeutic efficacy of cell therapy. Additionally, PET imaging allows the serial in vivo assessment of the inflammatory and immunological response by visualizing, for instance, a T-cell response [39]. Modulation of the immune response could provide additional benefit to combat tumor cells. The PET imaging is at present the only in vivo technology that can assess these various molecular aspects of stem cell efficacy and lead to a mechanistic understanding of how stem cells attack tumor cells.

Molecular imaging by PET is already used clinically to evaluate stem cell therapy for different neurological diseases besides the classical brain tumors [40]. Interesting in this context are patients with primary central nerve syndrome (CNS) lymphoma under the age of 60 years: high dose chemotherapy with autologous stem cell therapy represents one of the first-line treatment options in this subpopulation [41]. Additionally, simple FDG-PET leads to a $92 \%$ positive and $88 \%$ negative prediction of treatment outcome and correlates more strongly with disease-free survival in cerebral lymphoma than computed tomography [42]. The FDG-PET can therefore already serve an exquisite role in patient selection and help to determine which conditions are most suitable for stem cell therapy $[43,44]$. Serial quantitative PET will provide a means to monitor treatment progression [45] and afford a refinement of dosage and integration with other therapies.

Imaging of brain tumors already commonly uses FDG-PET. Translation of cell therapy for brain tumors will be best suited in conditions where imaging technology can already provide a robust baseline assessment that would allow patient selection. As the clinical translation of stem cell therapy for brain tumors progresses [45], preclinical validation studies will need to rely more on molecular imaging to ensure the safety and efficacy of this promising therapy. Growing sophistication of molecular imaging will allow increasingly sophisticated therapeutic approaches to be envisaged and monitored in vivo [46-49]. The multitude of therapeutic effects that can be exerted by unaltered and genetically engineered stem cells will need to be determined in terms of their efficacy in relation to the molecular composition of the tumor. Being able to select patients at an early stage based on a molecular signature of the entire tumor will allow clinicians to determine what strategy might be best suited to remove the neoplastic cells.

With increasing focus on the advance towards (more) curative solutions in brain tumor therapies, it is hard not to be excited by the potential of stem cell-based therapies. However, the efficacy of stem cell therapy continues to remain in question. Initial clinical trials have focused on evaluation of multiple adult stem cell phenotypes in their unaltered, native state as a "first generation" resource for repair. Though significant strides in perfecting delivery of these biologics to the diseased brain have been achieved, the benefits with regard to cerebral functional recovery have been modest at best. There is a clear need for robust genetic characterization before widely using the above described clinical stem cell therapy approaches. We have to be certain that the genome of the starting material is stable and normal, but the limited resolution of conventional karyotyping is unable to give us such assurance. Advanced molecular cytogenetic technologies and single nucleotide polymorphism analysis should be introduced [50]. As seen with pharmacotherapeutics in the last century, successful translation of "second generation" biotherapeutics in the $21^{\text {st }}$ century will require close integration of a community of practice and research to ensure broad application of this emerging technology in the treatment of brain tumors.

Developments in molecular imaging will therefore provide the diagnostic framework of a molec- 
ular medicine of brain tumors [12]. This is of special importance as, despite exciting initial reports, clinical potency of stem cell therapy in animal brain tumor models has to date proven disappointing. Attempts to extrapolate the animal study results to humans are stymied by the fact that stem cells are heterogeneous, resulting in differences in their efficacy. Indeed, therapeutic success relies on an effective strategy to select for a stem cell sub-population within some particular stage of the development at which they are competitive and capable of targeting brain tumors. Molecular imaging may help to better understand the specific tropic mechanisms that govern stem cell migration toward invasive tumors and the need to identify appropriate tissue sources and culture processes for the generation of adequate therapeutic stem cell populations. Despite all these current limitations, the use of stem cells as vectors for the treatment of brain tumors holds significant promise and may prove to be an important therapeutic modality for patients with malignant brain tumors. In combination with stem cells, molecular imaging may help to revolutionize a personalized, molecularly targeted medicine.

\section{References}

1. Schaller B. Neuroprotection in brain tumors: pathophysiological sense or nonsense? Nervenarzt 2003; 74: 1134-6.

2. Schaller BJ, Buchfelder M. Neuroprotection in primary brain tumors: sense or nonsense? Expert Rev Neurother 2006; 6: 723-30.

3. Park DH, Eve D. Regenerative medicine: advances in new methods and technologies. Med Sci Monit 2009; 15: RA 233-51.

4. Tampin P. Adult neurogenesis and the pathogenesis of Alzheimer's disease. Med Sci Monit 2009: 15: LE 1-1.

5. Alberti E, Los M, Garcia R, et al. Prolonged survival and expression of neural markers by bone marrow-derived stem cells transplanted interbrain lesions. Med Sci Monit 2009; 15: BR47-54.

6. Uyanikgil Y, Balcioglu H. Neural stem cell therapy in neurological diseases. Arch Med Sci 2009; 5: 296-302.

7. Schaller BJ, Cornelius JF, Sandu N, et al. Molecular imaging of brain tumors personal experience and review of the literature. Curr Mol Med 2008; 8: 711-2.

8. Arasho BD, Schaller B, Sandu N, et al. Gender-related differences in pituitary adenomas. Exp Clin Endocrinol Diabetes 2009; 117: 567-72.

9. Schaller B. Influences of brain tumor-associated pH changes and hypoxia on epileptogenesis. Acta Neurol Scand 2005; 111: 75-83.

10. Schaller B, Graf R. Cerebral ischemia and reperfusion: the pathophysiological concept as a basis for clinical therapy. J Cereb Blood Flow Metab 2004; 24: 357-71.

11. Waerzeggers $\mathrm{Y}$, Monfared P, Viel T, et al. Methods to monitor gene therapy with molecular imaging. Methods 2009; 48: 146-60.

12. Sandu N, Schaller B. Stem cell transplantation in brain tumors: a new field for molecular imaging. Mol Med 2010; 16: 433-37.
13. Li X, Liao D, Gong P, et al. Neural differentiation of periodontal ligament stem cells induced by neurotrophic Schwann cells factor. Arch Med Sci 2009; 3: 313-20.

14. Schaller B, Cornelius JF, Sandu N. Molecular medicine successes in neuroscience. Mol Med 2008; 14: 361-4.

15. Aboody K, Brown A, Rainov NG, et al. Neural stem cells display extensive tropism for pathology in adult brain: Evidence from intracranial gliomas. Proc Natl Acad Sci U S A 2000; 97: 12846-51.

16. Benedetti S, Pirola B, Pllo B, et al. Gene therapy of experimental brain tumors using neural progenitor cells. Nat Med 2000; 6: 447-50.

17. Schmidt NO, Przylecki W, Yang W, et al. Brain tumor tropism of transplanted human neural stem cells is induced by vascular endothelial growth factor. Neoplasia 2005; 7: 623-9.

18. Staflin K, Honeth G, Kalliomaki S, et al. Neural progenitor cell lines rat inhibit tumor growth in vivo. Cancer Res 2004; 64: 5347-54.

19. Ethesham M, Kabos P, Kabosova A, et al. The use of interleukin 12-secreting neural stem cells for the treatment of intracranial glioma. Cancer Res 2002; 62: 5657-63.

20. Fomchenko EI, Holland EC. Stem cells and brain cancer. Exp Cell Res 2005; 306: 323-9.

21. Modo M, Roberts TJ, Sandhu JK, Williams SCR. In vivo monitoring of cellular transplants by magnetic resonance imaging and positron emission tomography. Expert Opin Bio Ther 2004; 4: 145-55.

22. Schaller BJ, Modo M, Buchfelder M. Molecular imaging of brain tumors: a bridge between clinical and molecular medicine? Mol Imaging Biol 2007; 9: 60-71.

23. Capala J, Bouchelouche K. Molecular imaging of HER2positive breast cancer: a step toward an individualized "image and treat" strategy. Curr Opin Oncol 2010; 22: $559-66$.

24. Zlokovic BV, Apuzzo ML. Cellular and molecular neurosurgery: pathways from concept to reality. Part 1: Target disorders and concept approaches to gene therapy of the central nervous system. Neurosurgery 1997; 40: 789-803.

25. Modo M, Hoehn M, Bulte J. Cellular MR imaging. Mol Imaging 2005; 4: 1-21.

26. Schaller B. Usefulness of positron emission tomography in diagnosis and treatment follow-up of brain tumors. Neurobiol Dis 2004; 15: 437-48.

27. Cohen B, Ziv K, Plaks V, et al. MRI detection of transcriptional regulation of gene expression in transgenic mice. Nat Med 2007; 13: 498-503.

28. Genove G, DeMarco U, Xu H, et al. A new transgene reporter for in vivo magnetic resonance imaging. Nat Med 2005; 11: 450-4.

29. Schaller B. Positron emission tomography in neuroscience. An integrative part of clinical diagnostic methods and experimental research. Radiologie 2005; 45: 186-96.

30. Schaller B. Ischemic preconditioning as induction of ischemic tolerance after transient ischemic attacks in human brain: its clinical relevance. Neurosci Lett 2005; 377: 206-11.

31. Chin BB, Nakamoto Y, Bulte JW, et al. 111In oxine labeled mesenchymal stem cell SPET after intravenous administration in myocardial infarction. Nucl Med Commun 2003; 24: 1149-54.

32. Jacobs A, Braulich I, Graf R, et al. Quantitative kinetics of (124I)FIAU in cat and man. J Nucl Med 2001; 42: 467-75. 
33. Schaller B. State-of-the-art-imaging-methods to investigate the neurovascular mechanism in the origin of Alzheimer`s disease. Differential diagnostic evaluations to other types of dementia. Neuropsychiatr Dis Treat 2008; 4: 585-612.

34. Jacobs AH, Li H, Winkeler A, et al. PET-based molecular imaging in neuroscience. Eur J Nucl Med Mol Imaging 2003; 30: 1051-6.

35. Loimas S, Wahlfors J, Janne J. Herpes simplex virus thymidine kinase-green fluoresceint protein fusion gene: New tool for gene transfer studies and gene therapy. Biotechniques 1998; 24: 614-8.

36. Shah K, Hsich G, Breakefield XO. Neural precursor cells and their role in neuro-oncology. Dev Neurosci 2004; 26 : 118-30.

37. Matusik E, Wajgt A, Janowska J, et al. Cell adhesion molecular markers in ischaemic stroke patients: correlation with clinical outcome and comparison with primary autoimmune disease. Arch Med Sci 2009; 5: 182-9.

38. Schaller B. The role of endothelin in stroke: experimental data and underlying pathophysiology. Arch Med Sci 2006; 2: $146-58$

39. Koehne G, Doubrovin M, Doubrovina E, et al. Serial in vivo imaging of targeted migration of human HSV-TKtransduced antigen-specific lymphocytes. Nat Biotechnol 2003; 21: 405-13.

40. Kim SU, de Vellis J. Stem cell-based cell therapy in neurological diseases: a review. J Neurosci Res 2009; 87: 2183-200.

41. Colombat $P$, Lemevel A, Bertrand P, et al. High-dose chemotherapy with autologous stem cell transplantation as first-line therapy for primary CNS lymphoma in patients younger thant 60 years: a mulitcenter phase II study of the GOELAMS group. Bone Marrow Transplant 2006; 38: 417-20.

42. Filmont JE, Czernin J, Yap C, et al. Value of F-18fluorodeoxyglucose positron emission tomography for predicting the clinical outcome of patients with aggressive lymphoma prior to and after autologous stemcell transplantation. Chest 2003; 124: 608-13.

43. Becherer A, Mitterbauer M, Jaeger U, et al. Positron emission tomography with [18F]2-fluoro-D-2-deoxyglucose (FDG-PET) predicts relapse of malignant lymphoma after high-dose therapy with stem cell transplantation. Leukemia 2002; 16: 60-267.

44. Spaepen K, Stroobants S, Dupont P, et al. Prognostic value of pretransplantation positron emission tomography using fluorine 18-fluorodeoxyglucose in patients with aggressive lymphoma treated with high-dose chemotherapy and stem cell transplantation. Blood 2003; 102: 53-9.

45. Cremerius U, Fabry U, Wildberger JE, et al. Pre-transplant positron emission tomography (PET) using fluorine-18fluoro-deoxyglucose (FDG) predicts outcome in patients treated with high-dose chemotherapy and autologous stem cell transplantation for non-Hodgkins lymphoma. Bone Marrow Transplant 2002; 30: 103-11.

46. Su H, Forbes A, Gambhir SS, et al. Quantization of cell number by a positron emission tomography reporter gene strategy. Mol Imaging Biol 2004; 6: 139-48.

47. Brower V. Search and destroy: recent research exploits adult stem cells attraction to cancer. J Natl Cancer Inst 2005; 97: 414-6.

48. De Witte O, Lefranc F, Levivier $M$, et al. FDG-PET as a prognostic factor in high-grade astrocytoma. J Neurooncol 2000; 49: 157-63.
49. Padma MV, Said S, Jacobs M, et al. Prediction of pathology and survival by FDG PET in gliomas. J Neuro-oncol 2003; 64: 227-37.

50. Stephenson E, Oglivie CM, Patel H, et al. Safety paradigm: genetic evaluation of therapeutic grade human embryonic stem cells. J R Soc Interface 2010; 7 Suppl 6: S677-88. 